\title{
Are We DONE Yet? A corpus-based analysis of DONE as a sentence-boundary diagnostic in DGS
}

\author{
Elena Jahn, Gabriele Langer, Cornelia Loos \\ University of Hamburg \\ \{elena.jahn,gabriele.langer,cornelia.loos\}@uni-hamburg.de
}

\begin{abstract}
This paper looks at the lexemes DONE $\alpha$ and DONE $\beta$ in the Public DGS Corpus to find out whether they have a tendency to occur more frequently in sentence-final position than elsewhere and are therefore good cues for identifying sentence boundaries in German Sign Language (DGS). Since the Public DGS Corpus does not contain annotations of sentence boundaries or continuous syntactic tagging, we used time-aligned German translations as provisional sentential units. DONE $\alpha$ and DONE $\beta$ were selected since the literature suggests that they are best analyzed as right-peripheral heads. Additionally, we compared the distribution of DONE $\alpha$ to that of ALREADY $\alpha$, which served as a baseline. The results suggest that DONE $\alpha$ and $\operatorname{DONE} \beta$ indeed occur more often at the end of a translation tag than elsewhere in DGS. A more detailed analysis of a randomly selected subset of tokens of DONE $\alpha$ and DONE $\beta$ revealed that some of their functions are associated more strongly with sentential boundaries than others. Restrictive focus and discourse marker uses occur more consistently in the final position than, e.g., aspectual uses.
\end{abstract}

Keywords: German Sign Language, corpus linguistics, sentence boundaries, diagnostics, lexical semantics

\section{Introduction}

Sign linguists have long grappled with the issue of determining clause boundaries in signed languages (Crasborn 2007; Hodge 2014; Johnston and Schembri 2007; Tang and Lau 2012). The problem is especially relevant for working with large quantities of spontaneously produced language data as it occurs in signed and spoken corpora (for an overview on sentence segmentation issues in spontaneous speech, see for example Chafe (1985) and work by Auer $(2000,2010)$. Identifying sentential units is a crucial prerequisite for many kinds of linguistic analyses from determining basic constituent order or argument structure to identifying serial verb constructions, and it also has practical implications. Sentence-based translations, for instance, enhance corpus usability and accessibility.

For German Sign Language (DGS), the following diagnostics have been proposed for identifying sentence or clause boundaries: Hansen and Hessmann (2007) propose a prosodicpropositional approach in which sentential units are equated with propositions and identified via topics and predications about those topics. Based on these propositional units, the 
authors identify (non-)manual elements typically encountered at a boundary: the lexical signs NOW, PAST (often at the beginning of a sentence or larger textual unit), and DONE (often at the end of sentences), the palm-up gesture, and prosodic elements such as pauses, gaze direction, nods, and blinks. ${ }^{1}$ Herrmann (2010) identifies blinks and pauses as prosodic boundary markers in DGS in sentences elicited by means of a translation task and short dialogues. As she points out, however, prosodic and syntactic constituents are not necessarily isomorphic and in some cases, non-manuals may spread over the former rather than the latter. Loos (2018) reviews a number of syntactico-semantic diagnostics for clausehood in American Sign Language (ASL) and DGS and proposes among others a modified subject pronoun copy test to distinguish coordination from embedding and rightward wh-movement as a diagnostic for distinguishing finite from infinitival complement clauses.

However, there are issues with applying these criteria to large quantities of natural language data: Syntactic diagnostics often require eliciting additional grammaticality judgements, which is not feasible for large collections of signed texts. Propositional and prosodic annotations are helpful strategies for segmenting texts into units that correspond roughly to the syntactic notion of a sentence, yet their annotation is time-consuming and therefore usually likewise not feasible for larger collections of signed texts. Even on the assumption that these practical challenges are solvable, we are still faced with a lack of comprehensive theoretical guidelines for what constitutes a sentence in DGS. The present study takes as its point of departure the difficulties of using existing diagnostics to determine sentential boundaries in large collections of signed texts and proposes to examine as diagnostics annotations that are typically available in sign language corpora: manual signs and time-aligned translations.

Looking specifically at the Public DGS Corpus (Konrad, Hanke, Langer, Blanck, et al. 2020), we first note that it does not include systematic annotations of prosodic cues or continuous syntactic tagging that could be used for the identification of clause boundaries. What the annotations do include are sign-by-sign glosses and mouthings/mouth gestures as well as time-aligned German and English translations. These data can be mined for lexical cues that point to sentence boundaries, e.g. the signs PUT-ASIDE and DONE, or the palm-up gesture. Hansen and Hessmann (2007) observe that these elements consistently occur at sentence boundaries, where they structure discourse units. PUT-ASIDE, for instance, signals a narrative conclusion and thereby the end of a discourse unit. Given the low textual frequency of PUT-ASIDE in the Public DGS Corpus ( 110 tokens) and the at least partially gestural use of palm-up (Cooperrider, Abner, and Goldin-Meadow 2018), we focus on two lexemes with the core meaning 'done', DONE $\alpha$ and DONE $\beta$. Hansen and Hessmann (2007) report that over half of their DONE tokens occur at the end of sentences, which is not surprising given that the sign can head a wide range of right-headed projections, functioning as a lexical verb, a focus marker, or a discourse particle (discussed in Section 2). Proceeding from this observation, we test how often the lexemes DONE $\alpha$ and DONE $\beta$ occur at the end of sentence-like units and whether they could therefore be useful lexical cues for predicting sentential boundaries.

Specifically, we look at the distribution of the following lexemes in the Public DGS Corpus (illustrated in Figure 1): DONE1A, DONE1B, DONE2 (grouped as DONE $\alpha$ ), and DONE4 (henceforth $\mathrm{DONE} \beta) .^{2}$ For a more detailed rationale for the grouping, see Section 3.2. We compared these lexemes to ALREADY1A, ALREADY1B and ALREADY3 (grouped as ALREADY $\alpha$ ) as a control or baseline (see Section 2.4). ALREADY1A and ALREADY1 B have the same manual form as

1. The sign that is glossed as DONE by Hansen and Hessmann (2007) is referred to as DONE $\beta$ in our analysis.

2. DONE3A/B is not included in the analysis as it is a combination of DONE2 and DONE4 with a total of 33 tokens. The corpus also contains DONE5 (0 tokens) and DONE6 (7 tokens), which were excluded from analysis due to their low token frequency. 
DONE1A and DONE1B; ALREADY3 is manually identical to DONE2. ${ }^{3}$

We check whether DONE $\alpha$ and $\operatorname{DONE} \beta$ occur more frequently in sentence-final position than elsewhere in the sentence and whether they occur sentence-finally more often than ALREADY $\alpha$. We contrast DONE $\alpha$ and ALREADY $\alpha$ as they share the same manual form but are predicted to have a different range of functions and, consequently, a different syntactic distribution. In addition, we analyze the semantic/pragmatic functions of a subset of DONE $\alpha$ and DONE $\beta$ tokens to see whether some uses are more closely associated with the sentencefinal position.

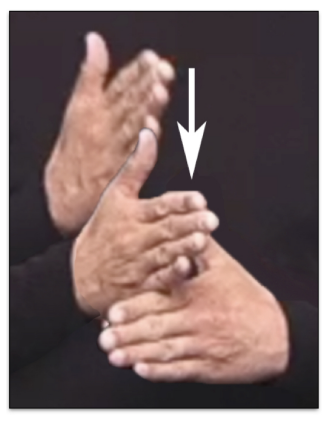

(a) DONE1A

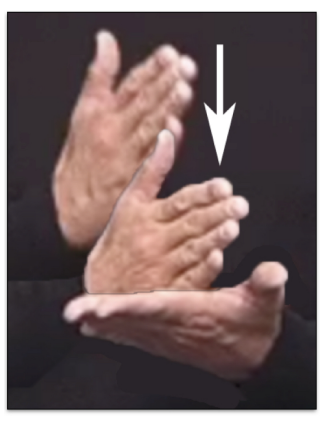

(b) DONE1B

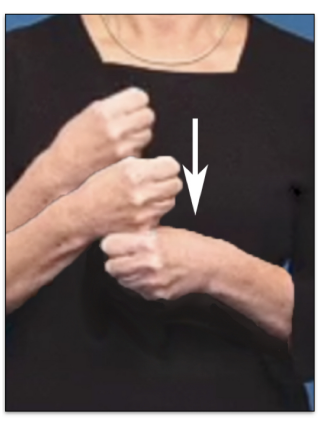

(c) DONE2

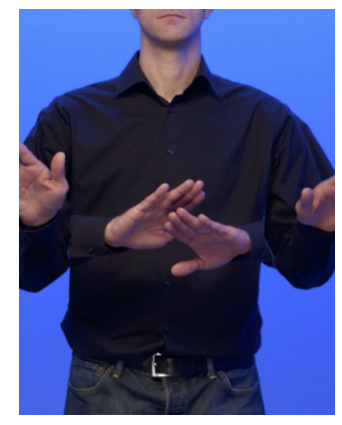

(d) DONE4

Figure 1: Lexemes

Since the corpus currently does not have syntactic or proposition-based marking of sentence boundaries, we operationalize time-aligned German translation tags as a provisional stand-in for DGS sentences (see Section 3.1.1 for a more detailed description of the annotation process).

The remainder of this paper is structured as follows: Section 2 discusses why DONE $\alpha$ and DONE $\beta$ are good candidates for identifying the right edge of a DGS sentence. In Section 3, we present our method, followed by the results in Section 4, which are discussed in Section 5.

\section{Background: Why DONE?}

Before we dive in, a quick note on the scope of the following overview: Most of the research literature focuses explicitly on DONE $\alpha$ (typically glossed FERTIG or, in English texts, FINISH) rather than on DONE $\beta$. Rathmann $(2003,2005)$ and Pfau and Steinbach $(2004)$ do not specify which lexeme DONE their claims are about. Our own analysis of the data suggests that both lexemes can be used in the functions described below, with the exception of the temporal connector/conjunction, which both in the literature and in a sample of our data set only occurs as DONE $\alpha$. Thus, bear in mind that the following discussion of DONE $\alpha$ in all likelihood also holds of $\operatorname{DONE} \beta$, unless otherwise indicated.

DGS is an SOV language in which not only the verb but also some projections at the extended IP and CP layers are right-headed. Higher CP strata relating to topic, focus, and sentence-typing have been claimed to be right-headed (Bross 2020), but their exponents are frequently non-manual (e.g. brow raising for topics and polar questions, brow furrowing for wh-questions) and have not been annotated systematically in the DGS Corpus. Fortunately, DONE $\alpha$ performs two functions that are located within this topographic region: It serves as a restrictive focus particle (Herrmann 2013) and, according to our data, as a discourse marker.

3. ALREADY and DONE were differentiated in the corpus by the different mouthings that usually accompany them. 
Based on the spreading behavior and intensity peak of non-manuals at the left periphery of the sentence, Bross (2020) suggests that the lower CP (speech act and speaker-oriented projections) is left-headed in DGS and we do not expect good candidates for right-peripheral elements here. Adverb placement in the higher IP is also predominantly pre-verbal, but several functional elements in the lower IP can occur post-verbally. This includes deontic and root modals (the latter prefer the post-verbal slot), success, conative, and terminative aspect markers (ACCOMPLISH 1C (MANAGE in Bross 2020),TRY1, and NOT-ANYMORE1A), the heads of manner phrases and, importantly, another function of DONE $\alpha$ : the perfective/completive aspect marker. Since most of the manual markers just mentioned are verbal heads, they are less fixed in their position than, e.g. adverbs and can also occur pre-verbally (e.g. modals, see Bross 2020; Proske et al. 2020). Most authors agree, however, that aspectual DONE $\alpha$ occurs exclusively clause-finally (but see Section 2.3 for discussion), which again makes this lexeme a good candidate for a lexical sentence-boundary indicator.

Before describing each of the right-peripheral uses of the 'done' lexemes in more detail, it bears noting that they have (at least) two additional uses, one of which is not expected to occur at sentence boundaries. First, the lexemes function as lexical predicates meaning '(to) end or finish, to complete', in which case they may be followed by one or more exponents of a right-headed functional category and are not expected to be sentence-final. Second, DONE $\alpha$ may serve as a temporal connector between events (sometimes followed by THEN), and may be developing into a temporal conjunction. Like its ASL counterpart FINISH, DONE $\alpha$ is frequently accompanied by brow raise in this function (see Janzen 1995 for ASL) and may originate from a subordinate temporal clause. Its prosodic independence from the surrounding sentences causes annotators to group it somewhat inconsistently as either the final element of one translation tag or the first element of the following. We now present a brief overview of the three main right-peripheral functions of DONE $\alpha$ and DONE $\beta$.

\subsection{Discourse marker}

Discourse markers structure complex interactional or discourse units and may consist of single words (e.g. well) as well as of multi-word expressions (e.g. $\left.y^{\prime} k n o w\right)$. They are typically located at the left or right periphery of their host utterance (Blühdorn, Foolen, and Loureda 2017) and are only weakly integrated into the syntactic and prosodic structure of an utterance (Imo 2017). In syntactic accounts of interactional language such as Wiltschko (2021), this distribution is accounted for by placing discourse markers that regulate turn-taking behavior at the outermost layer of syntactic structure.

To our knowledge, DONE $\alpha$ and DONE $\beta$ have not been described as discourse markers in the DGS literature (for an overview, see Volk and Herrmann 2021). Nonetheless, our data set contains tokens that function as information-structuring devices, serving e.g. as a turntaking signal not unlike English that's all (see, e.g. Norrick 2001). In example (1) from our data set (see here), DONE $\alpha$ (variant DONE1B) is used at the end of a narrative sequence during which the signer reports how she and her mother celebrated Christmas together one year. The signer ends her account with DONE 1 в and then looks towards the moderator and thereby turns away from the addressee, who subsequently launches the next turn.

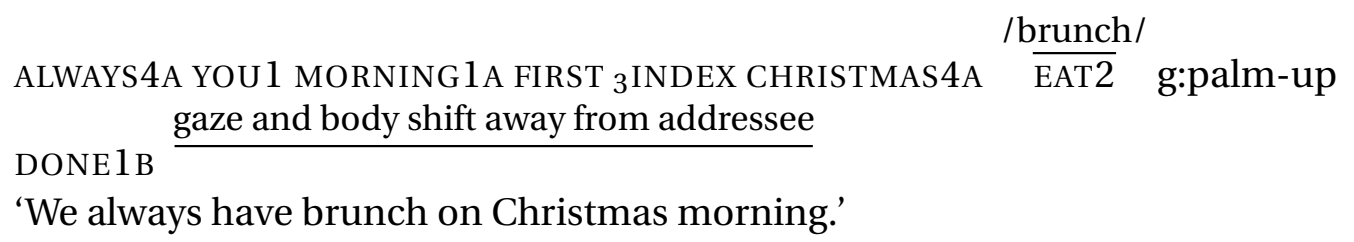




\subsection{Restrictive focus marker}

Another function of DONE $\alpha$ that is syntactically located within the CP layer is marking restrictive focus. Restrictive focus particles like English only are sensitive to focus, i.e. to new, important, or unexpected information. They look at the alternative set generated by focus and state that no other than the alternative mentioned in the sentence will make that sentence true. Hence, in Otto ate only carrots all week, the alternative set might contain potatoes and gelato and chicken wings, but only signals that none other than carrot makes the statement true. According to Herrmann (2013), DONE $\alpha$ serves this function in DGS either by itself or in conjunction with ONLY2A. Sentence (2) from our data set (see here) exemplifies this use for DONE $\beta$ (glossed DONE4). The signer discusses the lack of grammatical gender on English determiners and uses DONE $\beta$ to state that only the alternative T-H-E exists in the language.

(2) WORD2 KNOW2A YOU 1 D-E-R DIE D-A-S NONE2 T-H-E DONE4

'They have no words like 'der', 'die' or 'das', only 'the.'

Given its predominantly sentence-final occurrence and its likely grammaticalization from an aspectual head, Herrmann (2013) analyzes the restrictive focus marker DONE $\alpha$ as a functional head base-generated in $\mathrm{C}^{0}$.

\subsection{Aspect marker}

There is consensus in the DGS literature that DONE $\alpha$ performs aspectual functions (Pfau and Steinbach 2004; Rathmann 2005; Bross 2020) associated with perfective and/or completive marking. It portrays events as entities without internal structure that have been completed (Rathmann 2003). Our data set contained, for instance, sentence (3) (see here), in which DONE $\alpha$ (variant DONE1A) marks the completion of the changing event. There is less agreement on the position of aspectual DONE $\alpha$ : While it seems to occur predominantly in clausefinal position (Pfau and Steinbach 2004; Happ and Vorköper 2006; Rathmann 2005; Proske et al. 2020), pre-verbal uses have also been reported (Bross 2020). At this point it is unclear whether the pre-verbal position encodes a different aspect (e.g. the perfect), and if not, how the variable surface position should be modelled syntactically, but a left-branching perfective or completive head within the IP domain seems a reasonable assumption for clause-final DONE $\alpha$.

(3) CL:move-downwards INDEX $a$ TO-DRESS-UPPER-BODY1A DONE1A OUTSIDE CL:move 'I went downstairs to change and went outside when I was done.'

\subsection{Comparison with ALREADY}

One factor in determining how good of a predictor DONE $\alpha$ and DONE $\beta$ are in identifying the right boundary of a sentence is how often the lexemes occur sentence-finally in comparison to other lexemes that are not associated with right-peripheral functions. ALREADY $\alpha$ is a good candidate for comparison as it is manually identical with DONE $\alpha$ but in combination with the mouthing /schon/ 'already' performs a different range of functions. According to Bross (2020), ALREADY $\alpha$ marks anterior tense, that is, it introduces a presupposition that the event over which it scopes is located before a given reference time. In example (4) from the DGS Corpus (see here), ALREADY $\alpha$ (variant ALREADY1A) triggers the presupposition that the sign language festival has started before utterance time. Like other functional projections within 
higher IP strata, anterior tense seems to be concatenated left to right in DGS and therefore ALREADY $\alpha$ typically occurs to the left of the VP (Papaspyrou 2008).

\author{
/schon/ \\ NO2B THERE1 ALREADY1A BEGINNING1A \\ 'It [the sign language festival] had already started.'
}

As Bross (2020) points out, ALREADY $\alpha$ may have non-temporal functions such as marking the minimal element in a ranked scale of entities that will render a proposition true (e.g. schon and already in 32 Grad ist schon heiß, das ist nicht mehr nur warm ' 32 degrees is already hot, that's not only warm') (König 2017). Whether DGS ALREADY $\alpha$ may be used in this function and which syntactic position it would occupy still needs empirical investigation.

\title{
3 Method
}

\subsection{The corpus}

The DGS Corpus contains video data from 330 signers from different regions in Germany. Participants used DGS as their main language and were rooted in the signing community and in the region they represented. Participation was balanced for age, gender, and region (taking into consideration the population size of a given region, with a minimum of 16 participants per region). For more information, see Schulder et al. (2020).

Between 2010-2012, participants were filmed in pairs in mobile studio settings; data collection was facilitated by a deaf moderator who administered around 20 different elicitation tasks (more on elicitation methods in Nishio et al. 2010). Of the resulting 560 hours of filmed material, about 50 hours have been made available to the public and research community as the Public DGS Corpus. It contains over 355,000 tokens (as of Release 3) and serves as the basis for the current data set.

The medium of the signed texts is recorded video. Annotations are time-aligned to the video files and include German and English translations, glosses, and mouthings. The sign stream was tokenized and lemmatized manually by determining sign boundaries and matching each token to a lexical type (which may be a sign or one of its conventional uses). Each token is indicated by a time-aligned gloss.

\subsubsection{The translations}

German translations of the DGS texts were produced prior to lemmatization in order to support the annotators and provide access to the content of the signed texts. Translations were provided by external contractors, mostly professional sign language interpreters but in all cases individuals who were proficient in DGS and experienced in interpreting or translating. Many contractors were hearing, several were deaf or hard-of-hearing (Schulder et al. 2020). They were instructed to aim for a translation that is source-language oriented, which included, among other things, indicating when a DGS sentence was aborted (and thus closely representing the original text). The resulting texts contained relatively short German sentences. These German texts were then broken down into chunks including one or more sentences and time-aligned to the DGS texts by student annotators conversant in DGS. The annotators were instructed to ensure that the chunks of signing, to which a German translation was time-aligned, should form a semantically and prosodically coherent and intelligible utterance unit. With this aim in mind, annotators were encouraged to pay attention 
to prosodic boundary markers (head nods, body shift, gaze, and rhythm) as well as the occurrence of the palm-up gesture. Some German translations needed changes in sentence structure or sequence of elements to better fit assumed DGS structures and were re-arranged accordingly. Translations and appropriate chunking were checked again at several stages of the data preparation process (alignment, lemmatization, processing for publication, as well as a consistency check between glossing, annotations of mouthings, and German translations). A purely pragmatic step before publication was to make sure that the translation tags were short enough to serve as subtitles on the website www.meine-dgs.de. For a more detailed description of the annotation process, see Konrad, Hanke, Langer, König, et al. (2020). While translation tags were thus pragmatically determined and do not represent stringently theoretically-informed DGS sentence boundaries, we operationalize them as proxies for DGS sentences in lieu of a better alternative - the translations were geared towards a faithful representation of the source language DGS in terms of not only content but utterance structure, and they were created by DGS-competent translators.

\subsubsection{Glosses}

Spoken-language glosses are used as unique labels for signs in the DGS Corpus. ${ }^{4}$ The glosses are complemented by numbers and letters which indicate lexical and phonological variants (Konrad, Hanke, Langer, König, et al. 2020). The glossing system comprises two different levels of description: A type gloss represents the linguistic unit of a sign with all of its forms (including word forms and some phonological variants) and meanings and is marked by $\mathrm{a}^{\wedge}$ at the end of the gloss, e.g. END ${ }^{\wedge}$. The second level of description encompasses subsets of tokens that belong to one sign and that share a form variant and a conventionalized meaning. Given their shared meaning, grammatical behavior, and oftentimes shared mouthing, these lexicalized variants or subtypes are best characterized as lexemes for the purposes of this paper. Each subtype receives its own unique gloss whose glossword mnemonically indicates its core meaning. For example, the sign END $1^{\wedge}$ includes, among others, the lexemes ALREADY1 and DONE1, each of which has two phonological variants A and B. While identical in their manual form, ALREADY 1 and DONE 1 exhibit a non-identical set of meanings and functions, and they are further differentiated by their typical mouthing (/fertig/ vs. /schon/).

\subsection{Data set}

The queries and annotations for this study were conducted in the iLex annotation and working environment of the DGS-Korpus project. We only used data from the Public DGS Corpus in the extraction of the data set. ${ }^{5}$ From the subtype level we chose three groups of tokens for comparison: The first group, labelled DONE $\alpha$, consists of the tokens of three subtypes (DONE1A, DONE1B, and DONE2) that are considered phonological variants of each other for the purpose of this paper because they differ only in hand orientation (DONE1A vs. DONE1B) or in handshape (DONE1A vs. DONE2), and they do not show substantial differences in their meanings/uses. This group is contrasted with tokens of DONE4, which we refer to as DONE $\beta$,

4. Type and subtype glosses were assigned to tokens by student annotators conversant in DGS; this lemmatization process was reviewed by (near-)native signers of DGS working for the corpus project.

5. We opted to include only tokens from the Public DGS Corpus as its annotation quality is higher (it undergoes several checks before publication) and also because the published data is available for anyone to use for follow-up studies. We accessed the original data via the iLex database, whose content is subject to occasional changes due to corrections. This might result in small differences between our data set and the published version thereof in Release 3. 
a subtype of a different sign whose form differs substantially from DONE1A/1B/2 (DONE $\alpha$ ) but which displays similar meanings/uses. We test both groups for their alignment with the right boundary of a translation tag.

To arrive at an estimate for how well the lexemes DONE $\alpha$ and DONE $\beta$ fare in comparison to lexemes that are not predicted to be left-branching heads, DONE $\alpha$ is contrasted with a different conventional use of END $1^{\wedge}$, namely ALREADY $\alpha$. ALREADY $\alpha$ consists of two phonological variants, ALREADY1A and ALREADY1B, as well as of ALREADY3, which is assigned to a different sign but resembles DONE2 in form and displays the same meanings/uses as ALREADY1A and ALREADY1B.

The SQL query that generated our data set resulted in 2.673 tokens of the target lexemes DONE $\alpha$, DONE $\beta$ and ALREADY $\alpha$. For each token, the translation tag (TT) in which it occurred was extracted and analyzed separately. TTs with more than one token of the target lexemes were included more than once in the data set. We then excluded from analysis tokens that occurred in single-sign TTs (for which no distinct final vs. non-final position can be determined), tokens that occurred in aborted sentences, and TTs longer than 17 signs, which are considered extreme outliers (> $1.5 \mathrm{x}$ IQR) ${ }^{6}$ The resulting data set consists of 2.540 tokens of DONE $\alpha(n=1114)$, DONE $\beta(n=314)$, and ALREADY $(n=1112)$; the mean TT length is 7.41 signs.

For a subset of tokens of DONE $\alpha$ and $\operatorname{DONE} \beta$, we additionally annotated which function they perform to find out whether each function is equally likely to occur at the end of a translation tag. For this purpose, we identified TT positions of interest (final, penultimate, initial, and elsewhere) and randomly selected 174 TTs with a length of 4 to 10 signs for further analysis from our original data set. ${ }^{7}$ We annotated whether the lexemes were used as a) an aspect marker indicating that something was done thoroughly and to completion, b) a discourse marker indicating the end of a turn, a (part of a) story, or providing an evaluation, c) a restrictive focus marker meaning 'only', d) a lexical predicate meaning 'finish/complete an action', or e) a temporal or causal conjunction or clause. Concerning this last category, DONE $\alpha$ sometimes occurs in our data with non-manual topic marking and/or other nonmanuals that set it apart prosodically from the preceding and following utterances (e.g. via a pause or a torso shift). An example is shown in (5)(see here). Such data are compatible with an analysis of DONE $\alpha$ as the sole overt constituent of a temporal clause, e.g. 'after the judges finished watching', or as a temporal conjunction 'and then' emerging from this clausal use. We annotate such uses as 'temp' and remain agnostic as to their syntactic status.

TO-LOOK-AT 1 GOOD 1 TO-PLEASE1 TO-SIGN1A TO-SHOW1A brow raise, body lean forward

DONE1A LATER4 LAST1A TO-SEE1 INTERVIEW2

'The judges are watching to see whether or not they like it and then they interview everyone.'

An additional category 'other' captures lemmatization errors (e.g. NO3B for DONE $\beta$ ). Each of us annotated a subset of tokens and we discussed unclear cases. Tokens whose function could not be determined with certainty were labelled 'unclear'.

6. IQR (Interquartile Range) is a measure of dispersion that describes where the bulk of the data (the middle $50 \%$ of all values) are located. Anything above $1.5 \mathrm{x}$ IQR is considered an extreme outlier.

7. 130 tokens of DONE $\alpha$ and 44 tokens of DONE $\beta$ were included in the data sample. Although token selection was randomized, we ensured that the data sample represents the ratio of the lexemes under investigation by annotating $20 \%$ of the tokens in each TT position category for each phonological variant of DONE $\alpha$ and DONE $\beta$. 


\section{Results}

For each TT, we determined its length in number of signs and then noted the position of the target lexeme in this TT. Positions of target tokens were standardized by dividing their absolute position by the length of their respective TT. The results are shown in the graphs in Figure 2. In Figure $2 \mathrm{a}, 2 \mathrm{~b}$, and $2 \mathrm{c}$, the standardized position of DONE $\alpha$, DONE $\beta$, and ALREADY $\alpha$ are plotted on the $\mathrm{x}$-axis, with 1 corresponding to the last position. ${ }^{8}$ The number of tokens found in any given position is represented by the height of the bars on the y-axis. Figure $2 \mathrm{~d}$ shows the number of tokens for DONE $\alpha$ and DONE $\beta$ by positions in the TT grouped into categories: final, penultimate followed only by a gesture, penultimate, and elsewhere (any other position in the TT).

DONE $\alpha$ and DONE $\beta$ vs. ALREADY $\alpha$ : For both DONE $\alpha$ and DONE $\beta$, the final position has by far the highest token number. $31 \%$ (342/1114) of DONE $\alpha$ tokens occur in final position; and $52 \%$ $(162 / 314)$ of DONE $\beta$ tokens are TT-final. In contrast, the distribution of ALREADY $\alpha$ is more diffuse and we can identify several TT positions with similar or higher token frequencies than the final one. Only 6\% (62/1112) of ALREADY $\alpha$ tokens occur in final position. A chi square test confirms that the manually identical DONE $\alpha$ and ALREADY $\alpha$ differ in distribution: DONE $\alpha$ occurs significantly more frequently in TT-final position than ALREADY $\alpha\left(\mathrm{X}^{2}(1, \mathrm{~N}=\right.$ $2226)=236.5, \mathrm{p}<.001)$. The same holds for the formationally different DONE $\beta$ and ALREADY $\alpha$ : $\operatorname{DONE} \beta$ occurs significantly more frequently TT-finally than ALREADY $\alpha\left(\mathrm{X}^{2}(1, \mathrm{~N}=1426)=\right.$ $391.6, \mathrm{p}<.001)$.

DONE $\alpha$ vs. DONE $\beta$ : Both lexemes exhibit a higher preference for the final TT position than for any other position (see Figures 2a, 2b). This preference is even stronger when we include as TT-final those tokens that were only followed by a gesture or extra-linguistic material (e.g. palm-up, head nods, head scratching). Figure $2 \mathrm{~d}$ shows that 483 tokens or $43 \%$ of DONE $\alpha$ tokens are final or only followed by a gesture, and 187 tokens or $60 \%$ of DONE $\beta$. A chi square test revealed that DONE $\beta$ occurs more frequently in TT-final position than DONE $\alpha$ : $\left(\mathrm{X}^{2}(1, \mathrm{~N}\right.$ $=1429)=26.3, \mathrm{p}<.001)$.

Functions of DONE $\alpha$ and DONE $\beta$ by position: For the subset of 174 DONE $\alpha$ and DONE $\beta$ tokens, Table 1 lists their token frequencies by function and position within the TT. Penultimate tokens only followed by a gesture are counted as final, but we also indicate in parentheses what the numbers would be if penultimate tokens followed by a gesture were treated as penultimate.

We find that the 'done' lexemes occur most often at the end of a TT when they function as discourse markers $(19 / 26,77 \%)$ or as restrictive focus markers $(14 / 20,70 \%)$. Other functions occur less reliably at a TT boundary: temporal clauses/conjunctions occur TT-finally roughly half the time $(17 / 32,53 \%)$, and aspect markers even less so $(13 / 32,40 \%)$. As a lexical predicate, the 'done' lexemes occur at a TT boundary less than a third of the time $(33 / 47$, $30 \%)$.

8. While standardization was necessary to allow comparison across TTs of different length, it results in a TT length between 0 -1 where one position does not correspond to exactly one sign. 


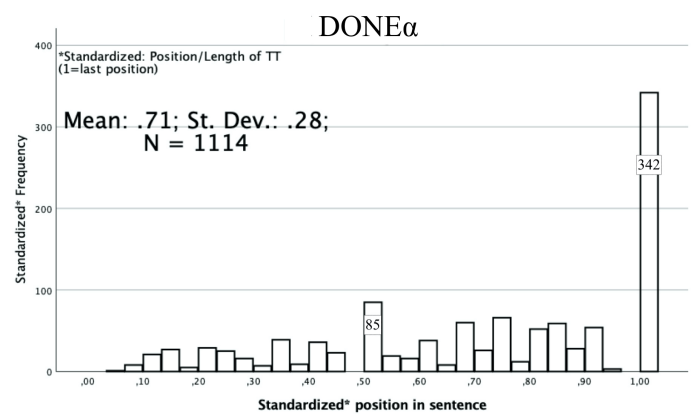

(a) DONE $\alpha$

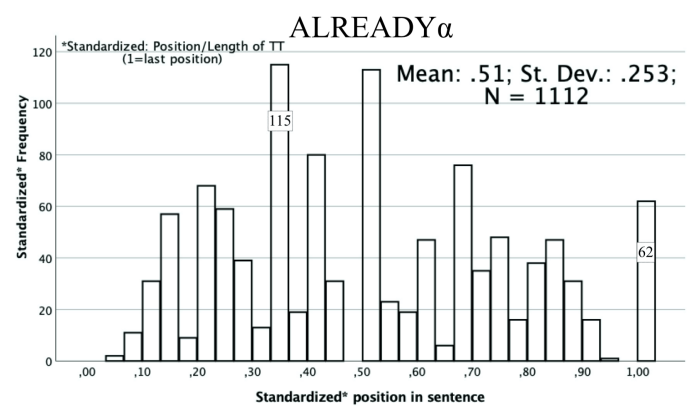

(c) ALREADY $\alpha$

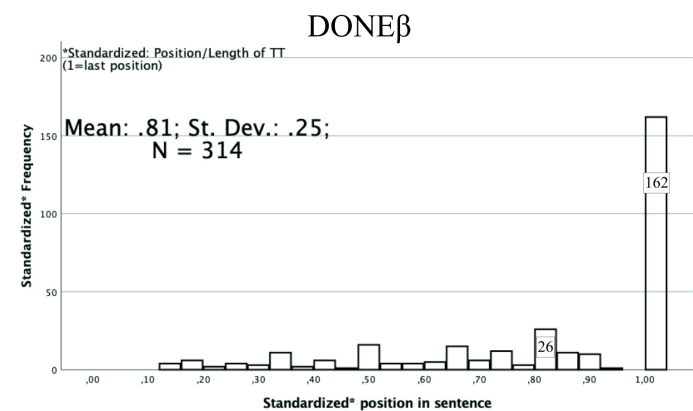

(b) DONE $\beta$

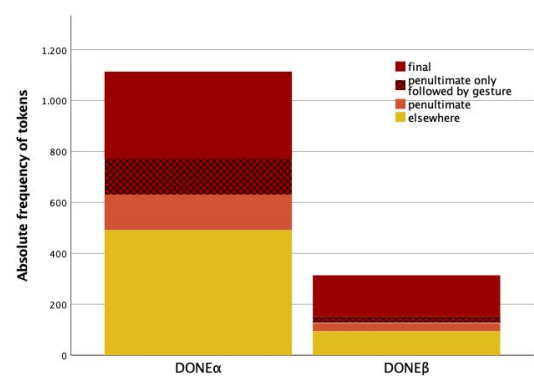

(d) DONE $\alpha, \beta$

Figure 2: Results

\begin{tabular}{||lccccc||}
\hline Functions & final & penultimate & elsewhere & initial & total \\
\hline aspect marker & $9(6)$ & $6(9)$ & 13 & 4 & 32 \\
discourse marker & $19(13)$ & $2(8)$ & 4 & 1 & 26 \\
focus marker & $14(10)$ & $0(4)$ & 6 & 0 & 20 \\
lexical predicate & $11(8)$ & $8(11)$ & 25 & 3 & 47 \\
temporal connector & 9 & 0 & 15 & 8 & 32 \\
other & $5(3)$ & $0(2)$ & 2 & 1 & 8 \\
unclear & $4(2)$ & $1(3)$ & 4 & 0 & 9 \\
\hline \hline total & $71(51)$ & $17(37)$ & 69 & 17 & 174 \\
\hline
\end{tabular}

Table 1: Functions of DONE $\alpha$ or DONE $\beta$ in different positions in the sentence.

\begin{tabular}{||lccc||}
\hline Functions & DONE $\alpha$ & DONE $\beta$ & total \\
\hline aspect marker & 28 & 4 & 32 \\
discourse marker & 12 & 14 & 26 \\
focus marker & 15 & 5 & 20 \\
lexical predicate & 36 & 11 & 47 \\
temporal connector & 31 & 1 & 32 \\
other & 1 & 7 & 8 \\
unclear & 7 & 2 & 9 \\
\hline \hline total & 130 & 44 & 174 \\
\hline
\end{tabular}

Table 2: Functions of DONE $\alpha$ or DONE $\beta$ grouped by lexemes. 
Table 2 shows how the different functions are distributed across the lexemes DONE $\alpha$ and DONE $\beta$. The numbers suggest that DONE $\beta$ is predominantly used as a discourse marker while the temporal clause/conjunction use is associated almost exclusively with DONE $\alpha$.

\section{Discussion \& conclusion}

How good of a cue is DonE? Provided that TTs approximate DGS sentences, we have shown that DONE $\alpha$ and DONE $\beta$ occur more frequently in sentence-final position than elsewhere, and that they occur significantly more frequently in this position than the control lexeme ALREADY $\alpha$. Of the two lexemes under investigation, DONE $\beta$ occurs sentence-finally significantly more often than DONE $\alpha$, but it also has a much lower token frequency in the Public DGS Corpus and therefore covers less of the data. If we disregard gestural and extra-linguistic material annotated in the corpus, the accuracy of using DONE $\alpha$ is $43 \%$ and that of DONE $\beta$ is $60 \%$. These odds could be improved by considering only discourse marking and restrictive focus marking uses of the 'done' lexemes, but that would essentially require part-of-speech tagging for the entire corpus. In the end, a combined method of lexical, propositional and prosodic cues might prove to be useful. Prosodic tagging might become more feasible in the future as automatic tagging of prosodic non-manuals improves.

New insights on DONE $\alpha$ and DONE $\beta$. Complementing existing research on DONE $\alpha$, we observe that DONE $\beta$ has a similar range of meanings and functions as DONE $\alpha$ but does not occur as a temporal connector in our sample annotations. There, it mainly functions as a discourse marker. To our knowledge, the discourse-marking functions of DONE $\alpha$ and DONE $\beta$ have not been well-described in the literature. Our analysis showed that both lexemes can signal the end of a turn or create cohesion between narrative or discourse segments. Further, $\operatorname{DONE} \beta$ may contribute modal meaning as an evaluative marker akin to the German modal particle einfach 'simply'. Our data sample contains eight uses of DONE $\beta$ which signal that the signer considers the content of their proposition to be an immutable fact that needs to be accepted as such and requires no further discussion. It is unlikely that this use is borrowed from German or constitutes a form of signed German, as tokens are not accompanied by the mouthing / einfach/ and, unlike their German counterpart, they occur in sentencefinal position. Regarding focus uses, we confirm Herrmann's (2013) observation that DONE $\alpha$ serves as a restrictive focus marker predominantly in sentence-final position and add that DONE $\beta$ may also perform this function. Turning to aspectual uses of DONE $\alpha$, we confirm Bross' (2020) observation that this use is not exclusively associated with the sentence-final position ( $40 \%$ of aspectual 'done' tokens are TT-final). Lastly, we observe an emerging use of DONE $\alpha$ as a temporal connector. Oftentimes, temporal uses of DONE $\alpha$ are offset prosodically from the surrounding utterances by brow raise and are variably grouped with a preceding or following TT; hence the connector use may derive from a temporal adverbial clause whose sole overt constituent is DONE $\alpha$.

A note on using TTs as sentential units. Given that we currently do not have theoretically informed clause or sentence boundaries in the DGS corpus, and in light of the fact that translators remained close to the structure of the source text and were proficient in DGS, we believe that TTs form a good first approximation to DGS sentences. Nonetheless, TT boundaries should be supplemented by prosodic cues such as pauses, nods, and blinks and cannot replace a theoretically informed and empirically consistent clause/sentence segmentation. 


\section{Acknowledgments}

This publication has been produced in the context of the joint research funding of the German Federal Government and Federal States in the Academies' Programme, with funding from the Federal Ministry of Education and Research and the Free and Hanseatic City of Hamburg. The Academies' Programme is coordinated by the Union of the German Academies of Sciences and Humanities.

\section{References}

Auer, Peter. 2000. "On line-Syntax-Oder: was es bedeuten könnte, die Zeitlichkeit der mündlichen Sprache ernst zu nehmen.” Sprache und Literatur 31 (1): 43-56.

_ 2010. “Zum Segmentierungsproblem in der Gesprochenen Sprache.” InLiSt - Interaction and Linguistic Structures 49.

Blühdorn, Hardarik, Ad Foolen, and Óscar Loureda. 2017. "Diskursmarker: BegriffsgeschichteTheorie-Beschreibung. Ein bibliographischer Überblick,” edited by Hardarik Blühdorn, Arnulf Deppermann, Henrike Helmer, and Thomas Spranz-Fogasy, 4-47. Diskursmarker im Deutschen. Reflexionen und Analysen. Göttingen: Verlag für Gesprächsforschung.

Bross, Fabian. 2020. The clausal syntax of German Sign Language: A cartographic approach. Language Science Press.

Chafe, Wallace. 1985. "Linguistic differences produced by differences between speaking and writing." In Literacy, language, and learning: The nature and consequences of reading and writing, edited by David R. Olson, Nance Torrance, and Angela Hildyard, 105-123. Cambridge: Cambridge University Press.

Cooperrider, Kensy, Natasha Abner, and Susan Goldin-Meadow. 2018. "The palm-up puzzle: Meanings and origins of a widespread form in gesture and sign." Frontiers in Communication 3:23.

Crasborn, Onno A. 2007. "How to recognise a sentence when you see one." Sign language \& linguistics 10 (2): 103-111.

Hansen, Martje, and Jens Hessmann. 2007. "Matching propositional content and formal markers: Sentence boundaries in a DGS text.” Sign Language \& Linguistics 10 (2): 145-175.

Happ, Daniela, and Marc-Oliver Vorköper. 2006. Deutsche Gebärdensprache: Ein Lehr-und Arbeitsbuch. Fachhochschulverlag.

Herrmann, Annika. 2010. "The interaction of eye blinks and other prosodic cues in German Sign Language." Sign Language \& Linguistics 13 (1): 3-39.

- 2013. Modal and focus particles in sign languages. De Gruyter Mouton.

Hodge, Gabrielle. 2014. "Patterns from a signed language corpus: Clause-like units in Auslan (Australian sign language).” PhD diss., Macquarie University.

Imo, Wolfgang. 2017. “Diskursmarker im gesprochenen und geschriebenen Deutsch.” In Diskursmarker im Deutschen. Reflexionen und Analysen, edited by Hardarik Blühdorn, Arnulf Deppermann, Henrike Helmer, and Thomas Spranz-Fogasy, 49-72. Göttingen: Verlag für Gesprächsforschung. 
Janzen, Terry. 1995. “The polygrammaticalization of FINISH in ASL.” Master's thesis, University of Manitoba.

Johnston, Trevor, and Adam Schembri. 2007. Australian Sign Language (Auslan): An introduction to sign language linguistics. Cambridge University Press.

König, Ekkehard. 2017. “Temporal and non-temporal uses of 'noch' and 'schon' in German.” In Actes du Colloque franco-allemand de Linguistique théorique, edited by Christian Rohrer, 181-203. Berlin: De Gruyter Mouton.

Konrad, Reiner, Thomas Hanke, Gabriele Langer, Dolly Blanck, Julian Bleicken, Ilona Hofmann, Olga Jeziorski, Lutz König, Susanne König, Rie Nishio, Anja Regen, Uta Salden, Sven Wagner, Satu Worseck, Oliver Böse, Elena Jahn, and Marc Schulder. 2020. MEINE DGS - annotiert. Öffentliches Korpus der Deutschen Gebärdensprache, 3. Release / MY DGS - annotated. Public Corpus of German Sign Language, 3rd release. Language Resource. V. 3.0. https://doi.org/10.25592/dgs.corpus-3.0.

Konrad, Reiner, Thomas Hanke, Gabriele Langer, Susanne König, Lutz König, Rie Nishio, and Anja Regen. 2020. Public DGS Corpus: Annotation Conventions / Öffentliches DGSKorpus: Annotationskonventionen. Project note. September. https:// doi.org/10.25592/ uhhfdm.1860.

Loos, Cornelia. 2018. "Detecting clauses and their dependencies in signed utterances: A syntacticosemantic approach.” Glossa: a journal of general linguistics 3 (1): 123.

Nishio, Rie, Sung-Eun Hong, Susanne König, Reiner Konrad, Gabriele Langer, Thomas Hanke, and Christian Rathmann. 2010. "Elicitation methods in the DGS (German Sign Language) corpus project." In Workshop on the Representation and Processing of Signed Languages, 178-185. Paris: ELRA.

Norrick, Neal R. 2001. “Discourse markers in oral narrative.” Journal of pragmatics 33 (6): 849-878.

Papaspyrou, Chrissostomos. 2008. Grammatik der deutschen Gebärdensprache aus der Sicht gehörloser Fachleute. Signum.

Pfau, Roland, and Markus Steinbach. 2004. On grammaticalization: Do sign languages follow the well-trodden paths. Poster presented at the Eighth International Conference on Theoretical Issues in Sign Language Research. Barcelona, Spain.

Proske, Sina, Derya Nuhbalaoglu, Annika Herrmann, Jana Hosemann, and Markus Steinbach. 2020. A Grammar of German Sign Language (DGS). SIGN-HUB Sign Language Grammars. https://doi.org/10.1075/sll.19011.wie.

Rathmann, Christian. 2003. "The optionality of agreement phrase: Evidence from German Sign Language (DGS)." In The role of agreement in natural language: Proceedings of the Fifth Annual Texas Linguistics Society Conference, edited by William Earl Griffin, 181-192. Austin, TX: Texas Linguistic Forum.

2005. Event structure in American Sign Language. The University of Texas at Austin.

Schulder, Marc, Dolly Blanck, Thomas Hanke, Ilona Hofmann, Sung-Eun Hong, Olga Jeziorski, Lutz König, Susanne König, Reiner Konrad, Gabriele Langer, Rie Nishio, and Christian Rathmann. 2020. Data Statement for the Public DGS Corpus. Project note. June. https: //doi.org/10.25592/uhhfdm.1855. 
Tang, Gladys, and Prudence Lau. 2012. “Coordination and subordination.” In Sign Language: An international handbook, edited by Roland Pfau, Markus Steinbach, and Bencie Woll, 340-365. Berlin: De Gruyter Mouton.

Volk, Elisabeth, and Annika Herrmann. 2021. "Discourse particles: Theoretical perspectives." In The Routledge Handbook of Theoretical and Experimental Sign Language Research, edited by Josep Quer, Roland Pfau, and Annika Herrmann, 480-499. London; New York: Routledge.

Wiltschko, Martina. 2021. The Grammar of Interactional Language. Cambridge: Cambridge University Press. 